\title{
Regenerative Endodontics for Upper Permanent Central Incisors after Traumatic Injury: Case Report with a 3-year Follow-up
}

Loai Alsofi

\begin{abstract}
Aim: The aim of this article is to describe a one-visit approach to attempt revascularization in the upper right and left central incisors after traumatic injury.

Background: A single-visit conservative revascularization approach can be used to promote root growth and maturation following traumatic dental injury and loss of pulpal tissues.

Case description: An eight-year-old female patient presented in Dentalia Clinics, Jeddah, Kingdom of Saudi Arabia, in 2016 with traumatic dental injury. Upon clinical and radiographic examination, it was found that the trauma resulted in loss of pulp vitality in two upper central incisors. Local anesthesia was administered, and a rubber dam was placed. Access cavity was done for each tooth separately. $2 \%$ chlorhexidine followed by sterile saline was used for irrigation with no instrumentation. Mineral trioxide aggregate (MTA) was used as the sealing material after blood clot formation. Follow-up showed continuous root maturation in the right central incisor, at 6 months, one year, two years, and three years. Root canal treatment was done in 2019 due to the development of periapical lesion. The 6-month follow-up radiograph also showed loss of the crown of the left central incisor due to a second trauma with retained apical root fragment. The fragment was embedded inside the bone and showed continuous maturation during the three years.

Conclusion: A single-visit regenerative endodontic approach showed successful results in revascularizing the upper permanent central incisor after loss of pulpal tissue.

Clinical significance: Immature permanent teeth can be treated by a single-visit revascularization procedure to promote root maturation and increase in radicular dentin length and width.

Keywords: Apical papilla stem cells, Dental pulp stem cells, Immature pulp, Regenerative endodontics.

The Journal of Contemporary Dental Practice (2019): 10.5005/jp-journals-10024-2618
\end{abstract}

\section{A IM}

The aim of this report is to describe a single-visit approach to attempt revascularization in the upper right and left central incisors after traumatic injury.

\section{BACKGROUND}

Root canal treatment of teeth with incomplete root formation has always been a challenge due to the difficulty to control root canal filling in open apices. Calcium hydroxide $\left(\mathrm{Ca}(\mathrm{OH})_{2}\right)$ apexification has been used as a treatment model for immature permanent teeth with incomplete root formation and open apex after the loss of pulp tissues. ${ }^{1,2}$ Mineral trioxide aggregate (MTA) can be used as an alternative in apexification, and it shows a higher survival rate than apexification in which $\mathrm{Ca}(\mathrm{OH})_{2}$ is used. ${ }^{3}$ Neither procedure can cause continuous root development, which leads to roots that are thin and prone to fracture. ${ }^{4,5}$ Recent evidence indicated better alternative methods to promote root maturation and apical closure. These methods aim to preserve the remaining dental pulp stem cells and mesenchymal stem cells of the apical papilla. The aim is to induce root formation by revascularization of the root canal. ${ }^{6}$

Stem cells of the apical papilla can survive pulp necrosis and can differentiate into secondary odontoblasts which can deposit dentin. ${ }^{6}$ The abundant blood supply to the apical papilla aids in the survival of these stem cells and their ability during revascularization procedures. It has also been suggested that some vital dental pulp stem cells in the apical part of the canal may survive partial pulp necrosis even in the presence of periapical pathology. ${ }^{4,6,7}$ These
Department of Endodontics, Faculty of Dentistry, King Abduaziz University, Jeddah, Kingdom of Saudi Arabia

Corresponding Author: Loai Alsofi, Department of Endodontics, Faculty of Dentistry, King Abduaziz University, Jeddah, Kingdom of Saudi Arabia, Phone: +966 555318481, e-mail: lalsofi@kau.edu.sa

How to cite this article: Alsofi L. Regenerative Endodontics for Upper Permanent Central Incisors after Traumatic Injury: Case Report with a 3-year Follow-up. J Contemp Dent Pract 2019;20(8):974-977.

Source of support: Nil

Conflict of interest: None

dental pulp stem cells have the potential to differentiate into odontoblast-like cells, which will aid in further root formation. ${ }^{8}$ It is pivotal for the success of the revascularization procedure to maintain the viability of dental pulp stem cells and stem cells of the apical papilla. That's why most case reports of revascularization procedures use minimal or no instrumentation at all to preserve these cells. ${ }^{4,9,10}$

A successfully revascularized tooth requires no further treatment assuming that healthy and vital tissues remain inside the canal with no periapical pathology. In contrast, apexification requires multiple visits to change calcium hydroxide until the apical barrier has formed and then MTA apical plug or gutta-percha root canal filling must be placed. ${ }^{11}$

In this report, we present a conservative single-visit revascularization technique using $2 \%$ chlorhexidine for irrigation followed by sterile saline with no instrumentation.

() The Author(s). 2019 Open Access This article is distributed under the terms of the Creative Commons Attribution 4.0 International License (https://creativecommons. org/licenses/by-nc/4.0/), which permits unrestricted use, distribution, and non-commercial reproduction in any medium, provided you give appropriate credit to the original author(s) and the source, provide a link to the Creative Commons license, and indicate if changes were made. The Creative Commons Public Domain Dedication waiver (http://creativecommons.org/publicdomain/zero/1.0/) applies to the data made available in this article, unless otherwise stated. 


\section{Case Description}

An eight-year-old female was referred in 2016 to the endodontic practice office at Dentalia Clinics, Jeddah, Kingdom of Saudi Arabia. The patient had a traumatic accident that occurred three months ago. The referral notes indicated intrusion of the upper left central incisor with grade 2 mobility and lateral luxation of the upper right central incisor with grade one mobility. Medical history was noncontributory. Dental history was nonsignificant other than the history of trauma to upper central incisors. Clinical examination did not reveal any teeth discoloration. Cold test was done, and it showed negative response in both teeth compared to the upper right and left lateral incisors. There was no tenderness to percussion or palpation. Gingival tissues were healthy with normal texture, and periodontal probing was within normal for all teeth. A periapical radiograph was taken (Fig. $1 A$ ), and it showed incomplete root development with parallel root walls in both teeth with no apparent periapical radiolucency. The radiograph showed very thin roots of both right and left central incisors. The initial diagnosis was pulp necrosis in both upper right and left central incisors. The treatment plan was to try revascularization of both teeth in an attempt to promote root maturation in length and width. The treatment plan was discussed with the patient's guardian. Potential risks, complications, and benefits of the suggested treatment were explained, and consent was signed. Teeth were splinted with wire and composite (Fig. 1B).

Two weeks later, the patient presented for the treatment. The treatment was carried out in a private dental office. Following local anesthesia administration using 3\% mepivacaine without a vasoconstrictor (Dentsply Pharmaceutical, York, PA), access cavity was done for each tooth one at a time using a dental operating microscope (Leica, Wetzlar, Germany). There were no signs for the presence of bleeding, pulpal tissues, or remnants of pulpal tissues. The diagnosis was confirmed as necrotic pulp with normal apical tissues. Due to the large size of the apex, no instrumentation was done. The coronal portion of the canal was then irrigated with $10 \mathrm{~mL}$ of $2 \%$ chlorhexidine gluconate (Vista Dental, Racine, WI, USA). The canals were then irrigated with sterile saline and dried with paper points. A size 10k file (Kerr, Romulus, MI, USA) was inserted into the root canal beyond the apex to induce bleeding. Pressure was applied using a dry cotton pellet to allow blood clot formation. The white MTA (Dentsply Tulsa Dental, Tulsa, OK, USA) was gently packed into the coronal part of the canal. A wet cotton pellet was carefully placed over MTA. The access cavities were sealed with Cavit temporary restorative material (Cavit ${ }^{\mathrm{TM}}, 3 \mathrm{M}$ ESPE, St Paul, MN, USA) (Fig. 1C). Two weeks later, the patient came to the clinic. There were no signs and symptoms of any disease in the area. After local anesthesia and rubber dam isolation, the temporary filling material and cotton pellet were removed from each tooth one at a time. It was verified that MTA was set. The access cavities were restored with bonded resin restorations (3M Filtek P60; 3M ESPE, St Paul, MN, USA).

\section{Follow-UP EXAMINATION}

The patient did not show up to the clinic until after six months, during which the patient had another trauma to the anterior region in which the upper left central incisor was fractured. Periapical radiograph was taken, and it showed that the crown of the upper left central incisor was lost, while the apical part of the root remained embedded inside the bone. The remaining root fragment continued to grow in length and width inside the bone (Fig. 2A). The radiograph also showed an increase in radicular dentin length and width with more apical closure in the upper right central incisor (Fig. 2B). At the two-year follow-up visit, a periapical radiograph was taken, and it showed almost complete root formation and closure of the apex of the upper right central incisor. The apical root fragment of the upper left central incisor also showed further maturation (Fig. 2C).

The patient presented in 2019, 3 years from the date of the revascularization procedure. A periapical radiograph was taken, and it showed complete root formation and apical closure of the upper right central incisor (Fig. 3A). It also showed periapical radiolucency related to the same tooth. It was explained to the parents that conventional root canal treatment is indicated at this time. Consent was obtained. After local anesthesia and rubber dam isolation, the composite filling was removed. Access to the root canal space was completed by removing MTA using ultrasonic tips under an operating microscope (Leica, Wetzlar, Germany). The canal was instrumented and irrigated with $6 \%$ sodium hypochlorite $(\mathrm{NaOCl})$. The canal was then dried and obturated using warm vertical compaction and thermoplasticized gutta-percha (B\&L Biotech, Fairfax, VA, USA) (Fig. 3B). A radiograph was taken to verify obturation quality, and then the access cavity was sealed by permanent restoration (Fig. 3C). Figure 3 shows the apical root
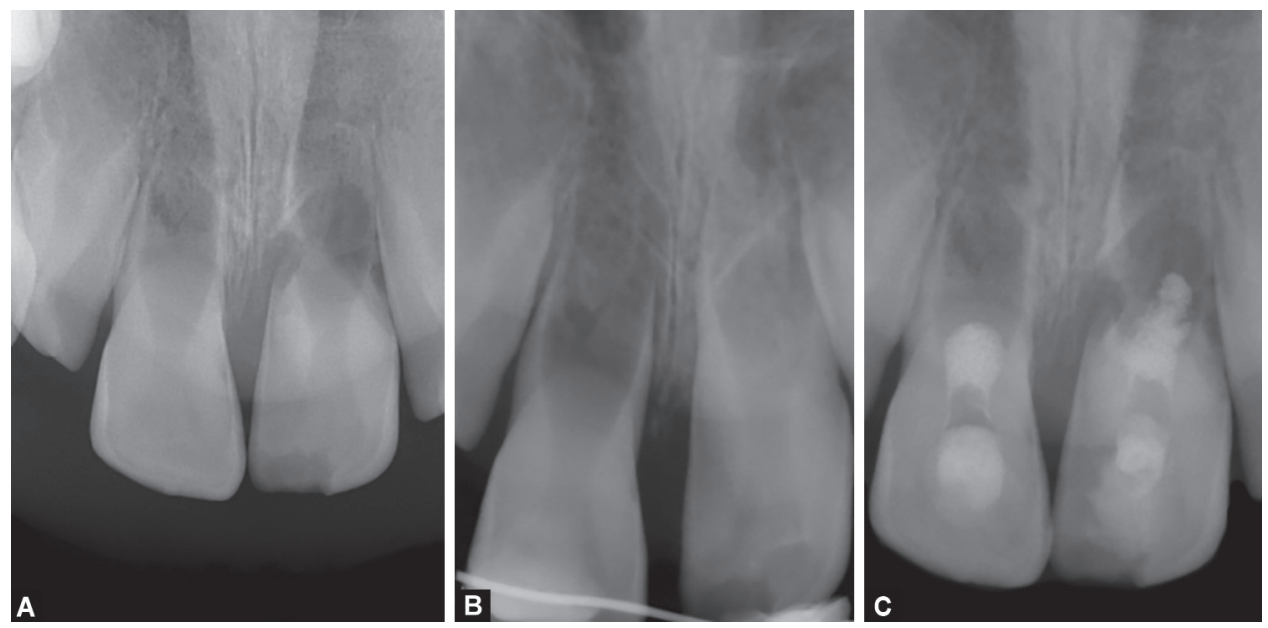

Figs $1 \mathrm{~A}$ to $\mathrm{C}$ : (A) Periapical radiograph of upper right and left central incisors. The radiograph shows incomplete root development in both teeth due to traumatic injury; (B) Teeth splinting using composite and wire; (C) MTA plug was placed in the coronal part of the canals of both teeth 

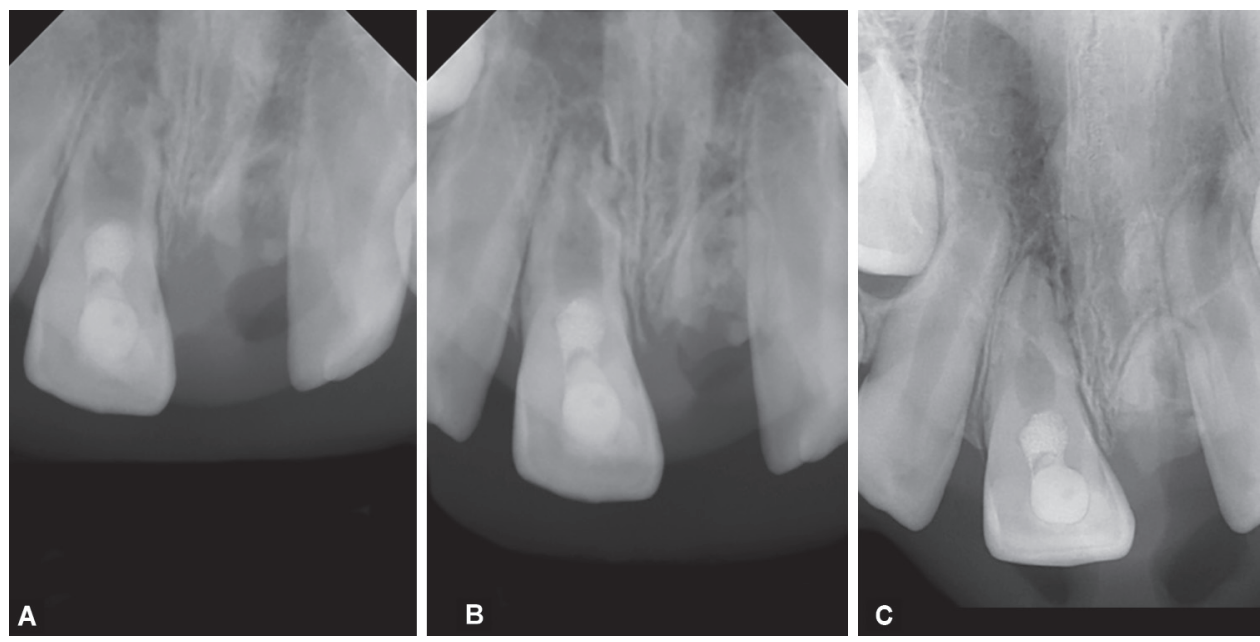

Figs 2A to C: (A) Six-month follow-up showing increase in dentin length and width in the upper right central incisor. It also shows crown fracture of the upper left central incisor with the apical part of the root still embedded in the bone; (B) One-year follow-up showing further increase in radicular dentin width and length and more apical closure; (C) Two-year follow-up periapical radiograph
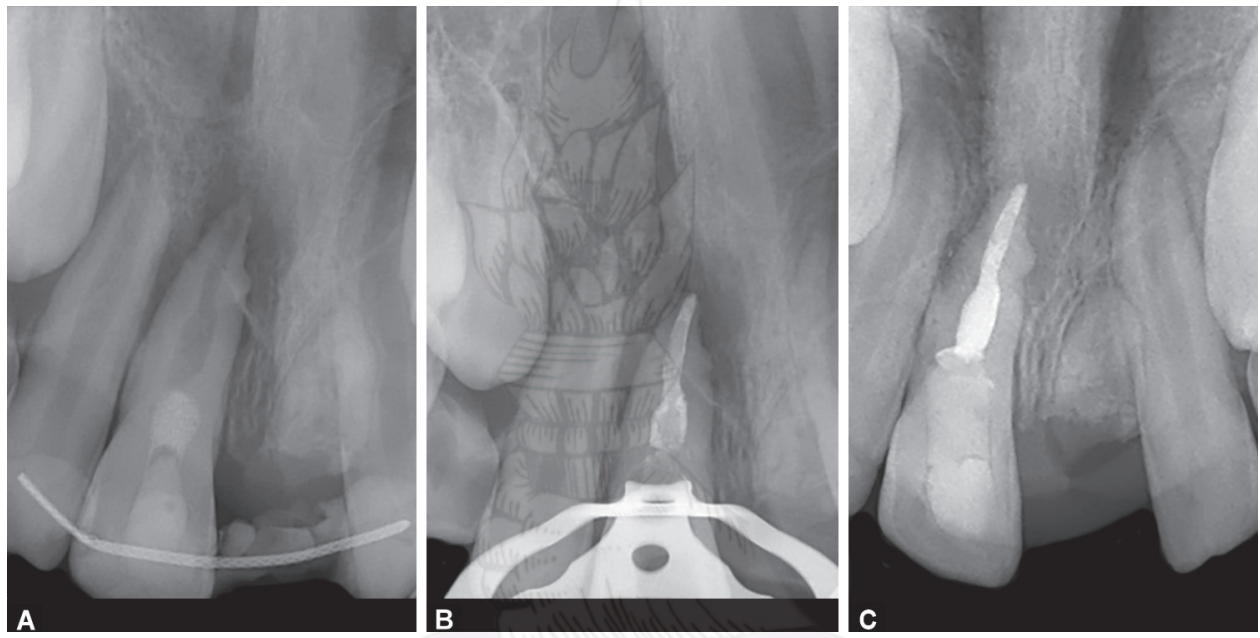

Figs 3A to C: (A) Three-year follow-up periapical radiograph shows periapical radiolucency related to the upper right central incisor; (B) Root canal treatment was done for the same tooth; (C) Final radiograph after the placement of final restoration

fragment of the upper left central incisor with a further increase in root length and width.

\section{Discussion}

Revascularization is a biologically-based regenerative treatment approach that allows root maturation by continuous deposition of dentin and cementum-like structure along dentin walls. The basics of revascularization were laid down in the early 1960s by Nygaard-Ostby and Hjortdal, ${ }^{12,13}$ but the concept comes from the literature of trauma cases. It was observed that revascularization occurred when avulsed teeth were replanted and when certain conditions were met. These conditions were the absence of bacterial challenge and the presence of a necrotic/ischemic root canal system that acts as a scaffold into which new tissues can grow through the large apical foramina. ${ }^{14}$

Revascularization procedure has been suggested as an alternative to apexification. It provides a superior outcome by continuation in root maturation and growth even in the presence of periapical pathology initially. ${ }^{5,9}$ This approach results in roots that are longer, thicker, and less susceptible to fracture. In this report, we used a modified single-visit technique in comparison to the original protocol described by Banchs and Trope. ${ }^{5}$ This less-invasive and conservative approach aims to minimize irritation of the remaining vital pulp tissues or stem cells of the dental pulp and apical papilla. Stem cells of the apical papilla are believed to be crucial for the success of revascularization procedures. ${ }^{6}$

Dental traumatic injuries result in disrupted blood supply to the pulp inside the tooth, which may result in pulp necrosis. The majority of dental injuries occur in children with intrusive luxation being the most common in the age group of 6-12 years. ${ }^{15}$ Intrusion represents a severe type of injury; the tooth is pushed axially into the socket resulting in damage to the alveolar bone, periodontal ligament (PDL), cementum, and pulp. Complications may follow, such as inflammatory root resorption, replacement resorption, and pulp necrosis. Although $97 \%$ of inflammatory resorption cases can be reversed by long-term calcium hydroxide treatment, ${ }^{16}$ there is no effective treatment for external replacement resorption. Luxation injuries result in rupture of the neurovascular supply at the apical foramen and the possibility of root fracture. ${ }^{17}$

The time frame for this report took three years since the date of initial examination until the final follow-up radiograph was taken. 
The possible risks and complications were considered as they were explained to the patient's guardian. Among these risks was the development of pulp necrosis after revascularization, which was managed by conventional root canal treatment. Another complication developed, which was crown fracture. The report indicated a favorable outcome of a one-step conservative revascularization procedure in cases of pulp necrosis due to traumatic injury, absence of infection inside the root canal, and absence of periradicular disease.

As follow-up to the healing progress in the upper right central incisor, we used the outcome criteria set by Bukhari et al. which include: complete healing, incomplete healing, and failure. ${ }^{18}$ The 6-month follow-up radiograph showed incomplete healing with the absence of clinical signs and symptoms, no periradicular disease, and an increase in dentin length and thickness. The one-year follow-up radiograph showed incomplete healing with progress in all of the previously mentioned features. The 2-year follow-up radiograph showed complete healing. The 3-year follow-up showed the development of periradicular radiolucency.

Regarding the root fragment of the upper left central incisor, the decision was to leave it inside the bone with continuous monitoring. The 6-month, one-year, two-year, and three-year follow-up radiographs indicated continuation in root maturation with an increase in length and width.

\section{CONCLUSION}

This three-year follow-up report showed growth and maturation of apical root fragments embedded in the bone after the loss of the crown. The report also showed complete root maturation followed by conventional root canal treatment for the upper central incisor.

\section{Clinical Significance}

The report shows that a single-visit conservative revascularization approach can be used as the treatment of choice for immature permanent teeth after loss of pulpal tissues.

\section{Acknowledgments}

The author denies any conflict of interest related to this case report.

\section{References}

1. Heithersay GS. Calcium hydroxide in the treatment of pulpless teeth with associated pathology. J Br Endod Soc 1975;8(2):74-93. DOI: 10.1111/j.1365-2591.1975.tb01000.x.

2. Frank AL. Therapy for the divergent pulpless tooth by continued apical formation. J Am Dent Assoc 1966;72(1):87-93. DOI: 10.14219/ jada.archive.1966.0017.
3. Jeeruphan T, Jantarat J, et al. Mahidol study 1: comparison of radiographic and survival outcomes of immature teeth treated with either regenerative endodontic or apexification methods: a retrospective study. J Endod 2012;38(10):1330-1336. DOI: 10.1016/ j.joen.2012.06.028.

4. Iwaya SI, Ikawa M, et al. Revascularization of an immature permanent tooth with apical periodontitis and sinus tract. Dent Traumatol 2001;17(4):185-187. DOI: 10.1034/j.1600-9657.2001.017004185.x.

5. Banchs F, Trope M. Revascularization of immature permanent teeth with apical periodontitis: new treatment protocol? J Endod 2004;30(4):196-200. DOI: 10.1097/00004770-200404000-00003.

6. Huang GT, Sonoyama W, et al. The hidden treasure in apical papilla: the potential role in pulp/dentin regeneration and bioroot engineering. J Endod 2008;34(6):645-651. DOI: 10.1016/j.joen.2008.03.001.

7. Lin L, Shovlin F, et al. Pulp biopsies from the teeth associated with periapical radiolucency. J Endod 1984;10(9):436-448. DOI: 10.1016/ S0099-2399(84)80266-6.

8. Shah N, Logani A, et al. Efficacy of revascularization to induce apexification/apexogensis in infected, nonvital, immature teeth: a pilot clinical study. J Endod 2008;34(8):919-925, discussion 1157. DOI: 10.1016/j.joen.2008.05.001.

9. Chueh LH, Huang GT. Immature teeth with periradicular periodontitis or abscess undergoing apexogenesis: a paradigm shift. J Endod 2006;32(12):1205-1213. DOI: 10.1016/j.joen.2006.07.010.

10. Jung IY, Lee SJ, et al. Biologically based treatment of immature permanent teeth with pulpal necrosis: a case series. J Endod 2008;34(7):876-887. DOI: 10.1016/j.joen.2008.03.023.

11. Rafter M. Apexification: a review. Dent Traumatol 2005;21(1):1-8. DOI: 10.1111/j.1600-9657.2004.00284.x.

12. Nygaard-Ostby B, Hjortdal O. Tissue formation in the root canal following pulp removal. Scand J Dent Res 1971;79(5):333-349. DOI: 10.1111/j.1600-0722.1971.tb02019.x.

13. Ostby BN. The role of the blood clot in endodontic therapy. An experimental histologic study. Acta Odontol Scand 1961;19:324-353. DOI: 10.3109/00016356109043395.

14. Kling $M$, Cvek $M$, et al. Rate and predictability of pulp revascularization in therapeutically reimplanted permanent incisors. Endod Dent Traumatol 1986;2(3):83-89. DOI: 10.1111/j.1600-9657.1986.tb00132.x.

15. Andreasen JO, Bakland LK, et al. Traumatic intrusion of permanent teeth. Part 1. An epidemiological study of 216 intruded permanent teeth. Dent Traumatol 2006;22(2):83-89. DOI: 10.1111/j.16009657.2006.00421.x.

16. Cvek M. Prognosis of luxated non-vital maxillary incisors treated with calcium hydroxide and filled with gutta-percha. A retrospective clinical study. Endod Dent Traumatol 1992;8(2):45-55. DOI: 10.1111/ j.1600-9657.1992.tb00228.x.

17. Andreasen FM. Pulpal healing after luxation injuries and root fracture in the permanent dentition. Endod Dent Traumatol 1989;5(3):111-131. DOI: 10.1111/j.1600-9657.1989.tb00348.x.

18. Bukhari S, Kohli MR, et al. Outcome of Revascularization Procedure: A Retrospective Case Series. J Endod 2016;42(12):1752-1759. DOI: 10.1016/j.joen.2016.06.021. 